\title{
Experiencia en capacitación de champions de la evidencia a través de E-learnings
}

\author{
Experience in Training Champions of the Evidence through \\ E-learnings
}

\section{Experiência no treinamento de campeões da evidência através de E-learnings}

\author{
Marcela Díaz-Fluhmann, Enf., MSc. * \\ Alejandra Belmar-Valdebenito, Enf., Diplm. **
}

\section{Resumen}

Introducción: La incorporación de Guías de Mejores Prácticas Clínicas de Registered Nurses' Association of Ontario, es reconocida como un invaluable aporte a la enfermería y así también se ha comprobado su contribución sin duda a la disminución de la variabilidad en la atención y seguridad de los pacientes. Es un reto incluir en los procesos de capacitación al personal de enfermería el uso de estrategias de aprendizaje E-learnings que permitan ampliar coberturas en la capacitación y obtener resultados adecuados. Objetivo: Presentar la experiencia y reflexiones respecto a la capacitación de champions de la evidencia, a través de la estrategia de aprendizaje Elearnings, en el personal de enfermería de la Clínica Las Condes de Chile sobre la implementación de guías de práctica clínica. Discusión y Conclusiones: Con base en la educación continua por fases, se tuvo en cuenta inicialmente la Guía de Práctica Clínica de Prevención de Úlceras por Presión y posteriormente la Guía de Práctica Clínica Registered Nurses' Association of Ontario de Manejo de accesos vasculares; se contó con la participación aproximada de 900 colaboradores, de los cuales son enfermeros cerca de 550. Se observó que después de la capacitación, haciendo uso de la plataforma E-learning de enseñanza, no hubo diferencias en el aprendizaje entre los grupos instruidos bajo metodología E-learning versus metodología presencial. [Díaz-Fluhmann M, BelmarValdebenito A. Experiencia en capacitación de champions de la evidencia a través de E-learnings. MedUNAB 2017; 20(2): 207-214].

Palabras clave: Enfermería Basada en la Evidencia; Capacitación Profesional; Práctica Clínica Basada en la Evidencia; Educación a Distancia; Aprendizaje.

\footnotetext{
* Enfermera, jefe servicio de Hemato-Oncología (2009-2015), Clínica Las Condes. Universidad Austral de Chile, Valdivia. Magíster en educación, mención informática educativa, Santiago de Chile, Chile.

** Enfermera, proyectos de docencia e investigación, Clínica Las Condes, Santiago de Chile, Chile. Universidad de los Andes, Santiago de Chile, Chile. Diplomada Cuidados Críticos Paciente Adulto, Diplomada Control de Infecciones asociada a la Atención de Salud, postítulo en Gestión y Administración de Empresas, postulante a grado de magísteren Epidemiología, Universidad de los Andes, Santiago de Chile, Chile.
}

Correspondencia: Alejandra Belmar Valdebenito. Clínica Las Condes, Santiago de Chile, Chile.E-mail:abelmar@clinicalascondes.cl 


\section{Abstract}

Introduction: The incorporation of Registered Nurses' Association of Ontario Best Practices Guidelines is recognized as an invaluable contribution to nursing, and its contribution to the reduction of variability in patient care and safety has also been proven. It is a challenge to include in nursing training processes the use of learning strategies "Elearnings" that allow expanding coverage in training and obtaining adequate results. Objective: To present the experience and reflections regarding the training of champions of the evidence in the nursing staff in "las Condes de Chile" Clinic on the implementation of clinical practice guidelines. Discussion and Conclusions: Based on continuous education by phases, the Clinical Practice Guideline for Pressure Ulcer Prevention was initially taken into account and subsequently the Clinical Practice Guideline Registered Nurses' Association of Ontario for the Management of Vascular Accesses. Approximately 900 employees attended and 550 of them were nurses. It was observed that after the training, using the E-learning teaching platform, there were no differences in learning between the groups instructed under the E-learning methodology versus the face-to-face methodology. [DíazFluhmann M, Belmar-Valdebenito A. Experience in Training Champions of the Evidence through E-learnings. MedUNAB 2017; 20(2): 207-214].

Keywords: Evidence-Based Nursing; Professional Training; Evidence-Based Practice; Education, Distance; Learning.

\section{Introducción}

Como es de público conocimiento, en 1854 Florence Nightingale introdujo medidas básicas de higiene y cuidados basados en sus observaciones, contribuyendo a la disminución de la morbi-mortalidad, convirtiéndose en la promotora del correcto diseño sanitario de los hospitales de la época. La visión de Nightingale fue el resultado de su experiencia durante la guerra de Crimea, donde junto a un grupo de enfermeras voluntarias entrenadas por ella, enfrentaron las duras y devastadoras condiciones sanitarias del Hospital Scutari. En este, los soldados heridos recibían tratamientos inadecuados por parte de un equipo sanitario muy escaso, con pésima higiene y altas tasas de enfermedades infecciosas como fiebre tifoidea, cólera y disentería. En muchos casos el resultado final era una muerte indigna de los pacientes (1).

Nightingale, aún hoy con sus aportes y su visión de futuro, sigue guiando a nuevas generaciones de enfermeras profesionales, orientándolas hacia el pensamiento crítico y al uso de la evidencia en las buenas prácticas de enfermería. En este sentido, referentes contemporáneos como la Doctora Doris Grinspun, han expresado su opinión al respecto, aseverando que; "Dentro de este contexto, es más urgente que nunca asegurar una práctica de enfermería, en todas sus

\section{Resumo}

Introdução: A incorporação das Diretrizes de melhores práticas clínicas da Associação de Enfermeiros Registrados de Ontário é reconhecida como uma contribuição inestimável para a enfermagem e sua contribuição para a redução da variabilidade nos cuidados e segurança do paciente. É um desafio incluir nos processos de treinamento para pessoal de enfermagem o uso de estratégias de aprendizado E-learning que permitem expandir coberturas no treinamento e obter resultados adequados. Objetivo: Apresentar a experiência e as reflexões sobre o treinamento de campeões da evidência, através da estratégia de aprendizado E-learning, na equipe de enfermagem da Clínica Las Condes do Chile sobre a implementação de diretrizes da prática clínica. Discussão e Conclusões: Com base na educação contínua por fases, a Diretriz de Prática Clínica para Prevenção de Úlcera de Pressão foi inicialmente levada em consideração e, posteriormente, a Diretriz de Prática Clínica Associação de Enfermeiros Registrados de Ontário para Gerenciamento de acessos vasculares; participaram aproximadamente 900 colaboradores, dos quais cerca de 550 são enfermeiros. Observou-se que, após o treinamento, utilizando a plataforma de ensino E-learning, não houve diferenças na aprendizagem entre os grupos instruídos sob a metodologia E-learning versus metodologia face-a-face. [DíazFluhmann M, Belmar-Valdebenito A. Experiência no treinamento de campeões da evidência através de E-learnings. MedUNAB 2017; 20(2): 207-214].

Palavras-chave: Enfermagen Baseada em Evidências; Capacitação Profissional; Prática Clínica Baseada em Evidências; Educação a Distância; Aprendizagem.

dimensiones: clínicas, administrativas, educativas, investigadoras y políticas, que estén finalmente basadas en la evidencia”(1). Además, la Dra. Grinspun señala que esta tarea debe ser tanto responsabilidad personal de cada enfermera/o como también del colectivo profesional dentro de las instituciones de salud.

Esta misma visión es la que mueve al hospital Clínica Las Condes (CLC), a la búsqueda de la evidencia en nuestro actuar como profesionales de la salud y es así como la incorporación de Guías de Mejores Prácticas Clínicas de Registered Nurses' Association of Ontario (RNAO) (2), se reconocen como un invaluable aporte a la enfermería especialmente por su contribución a la disminución de la variabilidad en la atención y seguridad de los pacientes.

CLC es un hospital privado en Santiago de Chile, fundado en el año 1982, que a la fecha cuenta con aproximadamente 310 camas y se encuentra actualmente en proceso de expansión. CLC atiende pacientes adultos, pediátricos y neonatales de todas las especialidades, tanto médicas como quirúrgicas, Es un centro de referencia nacional y latinoamericano en patologías de alta complejidad como trasplantes de órganos, cirugías de alta complejidad, cáncer y en especial manejo del paciente crítico en las unidades de cuidados intensivos (3). Por eso, este manuscrito pretende presentar la experiencia y reflexiones respecto a la 
capacitación de champions de la evidencia a través de la estrategia de aprendizaje E-learnings, en el personal de enfermería de Clínica Las Condes de Chile.

\section{Antecedentes}

Actualmente, CLC cuenta con un total aproximado de 3,700 empleados, de los cuales 610 son enfermeras y matronas. Debido a que precisamente es este grupo de profesionales quienes están más tiempo en la atención directa con el paciente hospitalizado, requieren de entrenamiento y capacitación continua en diversos conocimientos, técnicas y procedimientos.

Mantener las competencias laborales de enfermería es una necesidad que se concreta a través de programas de educación continua, realizados usualmente en el área de la salud en forma presencial, utilizando para ello los días libres de trabajo y en muchas ocasiones bajo condiciones desfavorables para el aprendizaje (4). Es por esto que el uso de las tecnologías de la información y comunicación da la posibilidad de mantener el nivel de aprendizaje, en comparación con la metodología tradicional presencial, permitiendo satisfacer la exigencia de mantener actualizados los conocimientos sin agobiar al personal sanitario.

En el año 2005 dentro de la Gerencia de Enfermería se desarrolla por primera vez un Software Educativo a través de una plataforma Web con el fin de incorporar la metodología E-Learning o educación a distancia vía Web, para todos los profesionales de enfermería de la institución. El primer curso E-learning correspondió a electrocardiografía básica. El personal capacitado evaluó esta dinámica como excelente, especialmente porque les da la posibilidad de desarrollar la capacitación desde su hogar y/o lugar de trabajo, según su disponibilidad de tiempo.

\section{Ventajas que promueve la Educación a Distancia (5):}

1. Supera los límites del aula.

2. Se adapta al ritmo de aprendizaje personal.

3. Permite un uso flexible del tiempo.

4. Emplea diversidad de medios y recursos.

5. No desvincula a las personas de su ámbito laboral o familiar.

6. Promueve la autonomía de los participantes.

\section{Beneficios del uso de Software Educativo en enseñanza-} aprendizaje (6):

Ventajas principales:

- Es un medio que ofrece a los alumnos información de forma visual a través del ordenador o computador

- Posee gran capacidad de motivación sobre los alumnos

- La comunicación puede ser bidireccional, estableciendo un feedback continuo con el alumno.

- El estudio puede adaptarse al trabajo y estilo de aprendizaje de cada alumno de forma individual, corroborándose el principio de individualización al que aboga el nuevo sistema educativo.

- La corrección y evaluación del ejercicio puede ser inmediata si hay interactividad.

- Es un medio capaz de mostrar y simular la realidad de forma casi perfecta.

Este modelo de experiencia ha continuado en la institución, modernizándose, por lo cual se ha convertido en pionera entre los hospitales del país. Hoy se puede decir que esta base educativa es la que abre las puertas a la difusión del conocimiento en este caso para participar en la creación de dos nuevos Cursos de Educación a distancia para el personal de enfermería basados en las Guías Clínicas de RNAO (7). Para ello institucionalmente se han seleccionado 2 Guías de Práctica Clínica RNAO para su implementación.

Las dos guías elegidas son:

1) Valoración del riesgo y prevención de las úlceras por presión $(8)$

2) Cuidados para reducir las complicaciones de los accesos vasculares(9)

La institución decide comenzar con la creación de metodología a distancia para la guía de prevención de las úlceras por presión.

\section{Proceso de implementación}

Para el desarrollo del proyecto educación a distancia en modalidad E-learning sobre guías clínicas, se genera en primera instancia el grupo de trabajo para implantación de guías de práctica clínica conformado por la enfermera de docencia, enfermeras de gestión de calidad y enfermera encargada del tratamiento de las heridas, el cual sesiona semanalmente y establece directrices para la implementación.

En estas sesiones se define que el curso E-learning sobre valoración del riesgo y prevención de las ulceras por presión, debe ser dirigido a todo el personal enfermero y matronas con labores clínicas, pero también administrativas, $\mathrm{y}$ a todo el personal técnico paramédico que cumpla las mismas características. Además, se decide que el personal de esta índole que pertenezca a las unidades de pabellones y urgencia, también realice el E-learning con el objetivo de aumentar la pesquisa temprana de lesiones por presión en los pacientes.

Finalmente, el curso es exigible para las siguientes unidades:

- Unidades de hospitalización critica adulto - pediátricos

- Unidades de hospitalización intermedia adulto pediátrica

- Neonatología

- Unidades de cuidados oncológicos

- Unidades de Médico Quirúrgico

- Unidades de Maternidad

- Pabellones

- Urgencia 
Luego de la definición del personal a capacitar, se realiza en nuestra Institución la primera sesión de Champions, con la participación de profesionales de enfermería y con representación de todos los servicios de hospitalización y pabellones quirúrgicos del establecimiento. En esta sesión se entrega copia de las guías a todas las participantes y se informa respecto del proyecto y sus fases de implantación (10) (Figura 1).

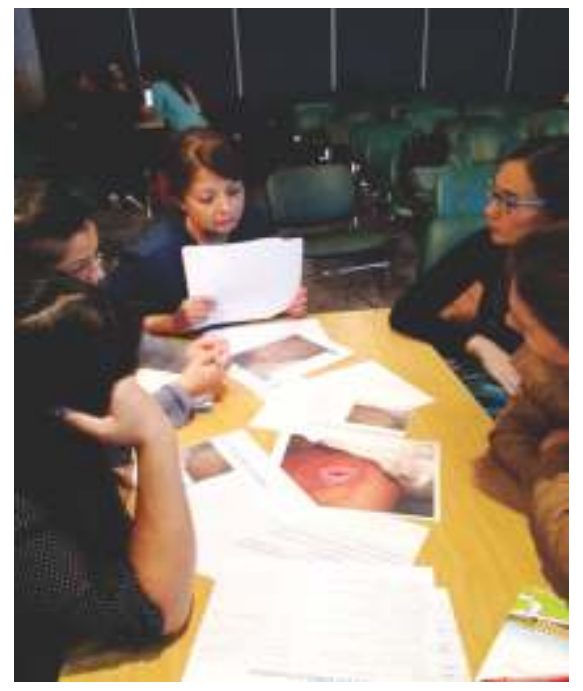

Fuente: Proporcionada desde base de datos proyecto RNAO.

Figura 1. Entrenamiento Champions personal de Enfermería.

\section{El proyecto se desarrolla en dos fases:}

Primera fase: Implementación de la Guía de Práctica Clínica de Prevención de Úlceras por Presión. Se definen contenidos y diseño del curso e-learning, el cual tendrá como característica el contar con la participación activa y permanente del personal de Enfermería, es decir, de aproximadamente 900 participantes.

Segunda fase: Corresponde a la aplicación de la Guía de Práctica Clínica RNAO de Manejo de accesos vasculares. Esta será solicitada para todo el personal de Enfermería, aproximadamente 550 participantes. Esta fase aún se encuentra en evaluación para definir su realización.

Para llevar a cabo el desarrollo de ambas fases, se obtiene financiamiento por parte de la Institución, en primera instancia, para el desarrollo del curso de E-learning de prevención de úlceras por presión basado en Guías RNAO dentro del marco del Programa de Capacitación del personal.

Los contenidos del programa e-learning se detallan en la Tabla 1, mientras que el proceso de realización del curso, se resumen en la Figura 2.

Aprendizajes esperados:

Al finalizar el curso los funcionarios serán capaces de:
- Valorizar el riesgo y la prevención de las úlceras por presión.

- Prevenir la formación de úlceras por presión.

- Disponer de técnicas de cuidado según el estadio de la úlcera.

\section{Evaluación}

Cada profesional que utilizó la plataforma E-learning de enseñanza, contó con una evaluación formativa de los contenidos teóricos entregados la cual fue realizada por todos los colaboradores al finalizar cada módulo del curso, exceptuando el módulo 7 que se incorpora como informativo (11). La metodología de evaluación consideró la utilización de los promedios de los puntajes de cada prueba más la evaluación de la participación en el curso, evidenciada por el número de accesos de los colaboradores a la plataforma elearning. Cabe señalar que, al incorporar contenidos nuevos relacionados con la implementación de las guías RNAO, el curso no posee una evaluación previa, solo cuenta con una prueba formativa al finalizar los contenidos, cuyo principal objetivo es guiar a los colaboradores hacia la correcta prevención de las úlceras por presión y la pesquisa temprana de lesiones.

Para aprobar el curso, el colaborador debía obtener una calificación de 90 puntos de un total de 150 posibles, conseguidos de la siguiente forma: 6 evaluaciones de 25 puntos cada una, de las que el colaborador deberá aprobar al menos 4 de ellas.

Es así como, después de la primera versión de este curso, se logra capacitar a un total de 941 colaboradores (Figura 3). De ellos, el 100\% logran la aprobación del E-learning (Tabla 2).

Mientras tanto, en el año 2015 se realiza capacitación de manera presencial sobre el mismo tema para todas las enfermeras y matronas de la institución con una duración de 3 horas y 30 minutos durante los meses de marzo, abril y septiembre. En este curso se retoma el tema sobre prevención de úlceras por presión y además se incorporan los primeros lineamientos sobre su tratamiento. Por último, se agrega un breve taller práctico para demostración del uso de insumos de prevención y una estación de trabajo para evaluación de casos clínicos.

Este curso es evaluado con una prueba teórica de 14 preguntas con alternativas simples. Los requisitos de aprobación del curso es obtener una nota 4.0 en la evaluación teórica. El programa educativo de las sesiones realizadas durante el mes de septiembre de 2015 (Figura 4).

Durante estas sesiones se capacita a un total de 143 colaboradores entre enfermeras y matronas, logrando la aprobación del curso en un $100 \%$ al igual que lo ocurrido con la metodología E-learning. 
Tabla 1. Contenidos del programa E-learning en prevención de úlceras por presión

\begin{tabular}{|c|c|}
\hline $\begin{array}{l}\text { Clase 1: } \\
\text { "Introducción a las } \\
\text { guías clínicas } \\
\text { RNAO aplicadas al } \\
\text { manejo de úlceras } \\
\text { por presión" }\end{array}$ & $\begin{array}{l}\text { 1.1 Responsabilidad en el manejo de las Guías. } \\
\text { 1.2 Objetivos del curso. } \\
\text { 1.3 Valoración de la piel y los factores de riesgo. } \\
\text { 1.4 Epidemiología } \\
\text { 1.5 La etiología y factores de riesgo de las úlceras por presión. } \\
\text { 1.6 Mecanismos que desencadenan las úlceras por presión (UPP) } \\
\text { 1.5 Clasificación por factores de las úlceras por presión. }\end{array}$ \\
\hline $\begin{array}{l}\text { Clase 2: } \\
\text { "Valoración del } \\
\text { riesgo y } \\
\text { prevención de las } \\
\text { úlceras por } \\
\text { presión. Parte 1” }\end{array}$ & $\begin{array}{l}\text { 2.1 Valoración de la piel al ingreso del paciente. } \\
2.2 \text { Inspección de la piel y factores de riesgo. } \\
2.3 \text { Zonas de riesgo. } \\
2.4 \text { Mecanismos de Producción. } \\
2.5 \text { Fuerzas de presión. } \\
2.6 \text { Clasificación de las úlceras. } \\
2.7 \text { Estadios. }\end{array}$ \\
\hline $\begin{array}{l}\text { Clase 3: } \\
\text { "Valoración del } \\
\text { riesgo y } \\
\text { prevención de las } \\
\text { úlceras por } \\
\text { presión. Parte 2" }\end{array}$ & $\begin{array}{l}\text { 3.1 Interpretación de la evidencia. } \\
\text { 3.2 El uso de herramientas de valoración, para la predicción del riesgo de úlceras por presión. } \\
\text { 3.3 Categorías de valoración del riesgo para determinar riesgos específicos y garantizar una } \\
\text { planificación de cuidados eficaz. } \\
\text { 3.4 Recomendaciones para la práctica basadas en la evidencia. } \\
\text { 3.5 Escala de valoración de riesgo de úlceras por presión (EVRUPP) de Braden. } \\
\text { 3.6 Riesgos e intervenciones asociadas. } \\
\text { 3.7 Mecanismo de elaboración e implantación de un programa personalizado de cuidados al paciente. }\end{array}$ \\
\hline $\begin{array}{l}\text { Clase 4: } \\
\text { "Valoración del } \\
\text { riesgo y } \\
\text { prevención de las } \\
\text { úlceras por } \\
\text { presión. Parte 3" }\end{array}$ & $\begin{array}{l}\text { 4.1 Recomendaciones para la práctica basadas en la evidencia según la etapa de intervención. } \\
\text { 4.2 Paciente sentado. } \\
\text { 4.3 Protección de la piel e incontinencia. } \\
\text { 4.4 Alimentación. } \\
\text { 4.5 Cuidados en la movilización }\end{array}$ \\
\hline $\begin{array}{l}\text { Clase 5: } \\
\text { "Recomendaciones } \\
\text { para la práctica. } \\
\text { Pabellones } \\
\text { Quirúrgicos" }\end{array}$ & $\begin{array}{l}\text { 5.1. Los riesgos de lesión en el paciente quirúrgico. } \\
\text { 5.2. Las posiciones quirúrgicas básicas y los puntos de apoyo de riesgo en cada una de ellas. } \\
\text { 5.3 Población de mayor riesgo y los factores quirúrgicos que influyen en el riesgo. } \\
\text { 5.4 Dispositivos para protección. } \\
\text { 5.5 Evidencia existente sobre la valoración del riesgo y prevención de las úlceras por presión en } \\
\text { pabellón. } \\
\text { 5.6 Tipos de lesiones. } \\
\text { 5.7 Recomendaciones para la práctica. } \\
\text { 5.8 Discusión de la evidencia. } \\
\text { 5.9 Medidas para la protección en pabellón. } \\
\text { 5.10 Dispositivos. } \\
\text { 5.11 Recomendaciones en posiciones quirúrgicas. }\end{array}$ \\
\hline $\begin{array}{l}\text { Clase 6: } \\
\text { "Recomendaciones } \\
\text { para la práctica. } \\
\text { Pediatría y } \\
\text { Neonatología" }\end{array}$ & $\begin{array}{l}\text { 6.1 Frecuencia y localización de UPP en pacientes pediátricos y neonatológicos. } \\
\text { 6.2 Escala de valoración de riesgo de UPP BRADEN Q para pacientes pediátricos menores de } 5 \text { años. } \\
\text { 6.3 Escala de Braden Q y sus definiciones. } \\
\text { 6.4 Detalle de cada ítem de la escala de NSRAS para la clasificación de los pacientes neonatales según } \\
\text { riesgo. } \\
\text { 6.5 Relación puntaje d e la Escala de valoración de riesgo de úlceras por presión en neonatología } \\
\text { NSRAS, y las intervenciones de enfermería. }\end{array}$ \\
\hline $\begin{array}{l}\text { Clase 7: "Guía } \\
\text { Clinica" }\end{array}$ & $\begin{array}{l}\text { 7.1. Sentido de la Guía. } \\
\text { 7.2. Motivo por el cual la Guía es importante. } \\
\text { 7.3. Análisis de la guía con fines de revisión ante una auditoría. }\end{array}$ \\
\hline
\end{tabular}

Fuente: Programa "Curso de valoración del riesgo y prevención de las ulceras por presión", Gerencia de Enfermería, 2014 


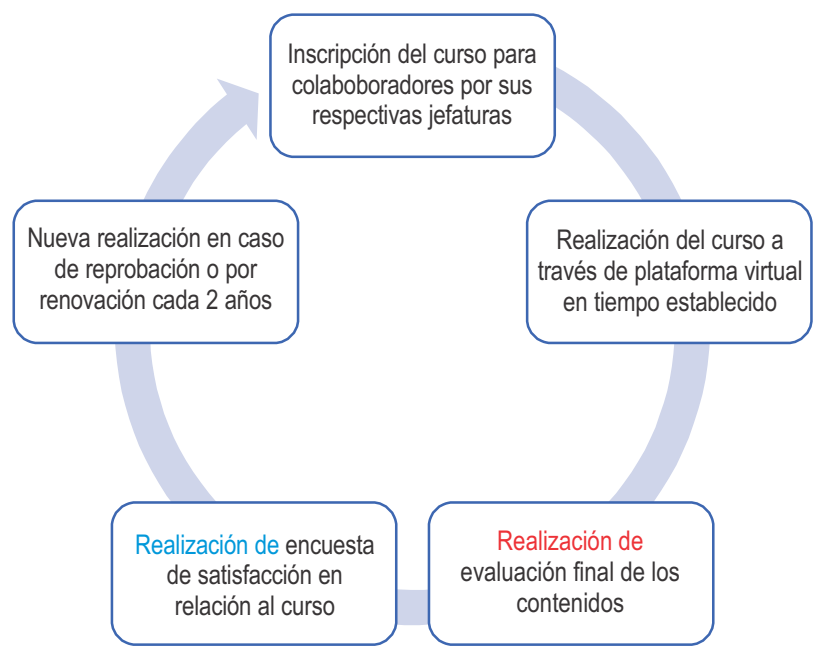

Fuente: Elaboración de los autores

Figura 2. Proceso de realización del curso E-learning.

Número de personal capacitado E-learning por estamento Años 2014 -2016 (n=941)

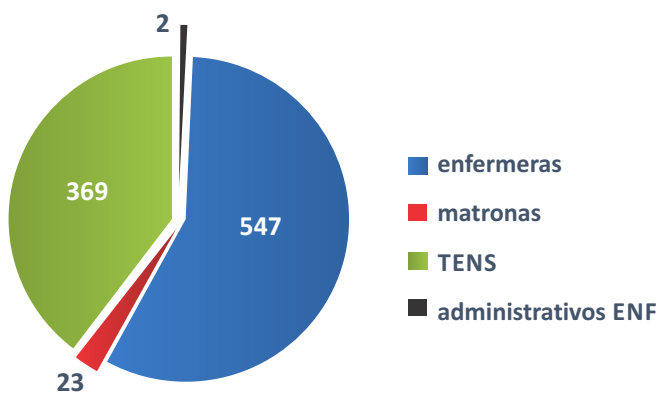

Fuente: Base de datos guía prevención de úlceras por presión RNAO. Años 2014-2016.

Figura 3. Total de personal capacitado por metodología E-Learning en prevención de ulceras por presión para los años 2015-2016.

Tabla 2. Total de personal con curso aprobado por metodología E-learning en prevención de ulceras por presión

\begin{tabular}{lc}
\hline Colaboradores con curso realizado & Número de colaboradores con curso aprobado \\
\hline Administrativo enfermería & 2 \\
\hline Auxiliar de enfermería & 369 \\
\hline Enfermera coordinadora & 13 \\
\hline Enfermera jefe departamento enfe rmería & 7 \\
\hline Enfermera(o) docente & 6 \\
\hline Enfermera(o) residente & 6 \\
\hline Enfermera/matrona & 515 \\
\hline Matrona & 22 \\
\hline Matrona jefe de departamento & 1 \\
\hline Total & 941 \\
\hline
\end{tabular}

Fuente: Programa “Curso de valoración del riesgo y prevención de las ulceras por presión”, Gerencia de Enfermería, 2015-2016 

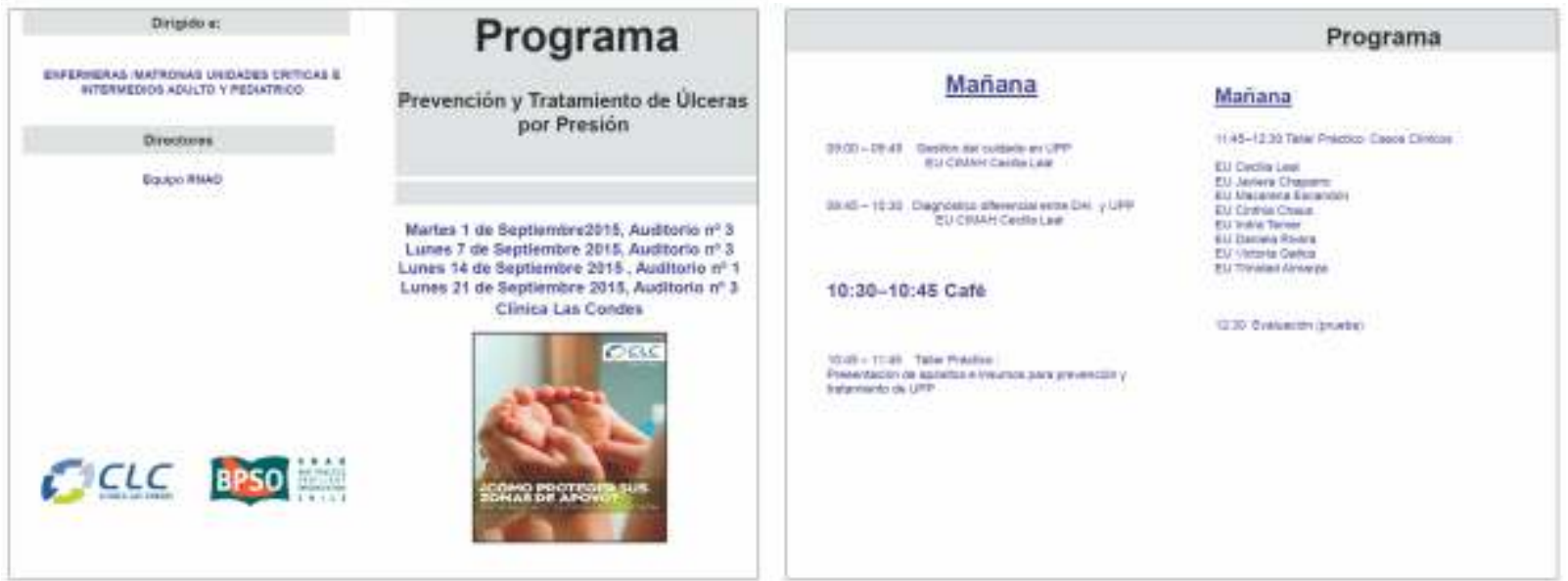

Fuente: Programa "Curso de valoración del riesgo y prevención de las ulceras por presión”, Gerencia de Enfermería, 2015.

Figura 4. Programa educativo por sesiones realizadas de prevención y tratamiento de úlceras por presión.

En cuanto a los resultados posteriores a la capacitación por medio de estas dos metodologías, se observa que la incidencia de úlceras por presión no se incrementa ni disminuye significativamente entre los grupos educados bajo metodología E-learning versus metodología presencial.

Esto se evidencia a través de los resultados en cuanto a la prevención y pesquisa temprana de las ulceras por presión entre los años 2014-2016 (Figura 5).

Cabe destacar que, a fines del año 2014, Clínica las Condes realiza la apertura de su nuevo edificio, el cual dobla la capacidad de las camas de cuidados intensivos y de cuidados intermedios adultos, lo que a su vez se traduce en un aumento de los días cama utilizados por los pacientes y en un aumento de la dotación de personal para estas unidades. Por consiguiente, el número de reportes del evento úlcera por presión aumentó momentáneamente durante el año 2015, pero su tendencia vuelve a la baja desde el año 2016, es por esta razón que el valor de la tasa se reduce, en conjunto con el valor absoluto del evento.
La capacitación al personal inicia en el año 2014 y se mantiene durante los años siguientes, requerida para todo el personal de enfermería que ingrese a la institución y se debe renovar cada 2 años de manera obligatoria.

\section{Conclusiones}

En resumen, no se observan resultados diferentes en la incidencia de úlceras por presión producto de la atención brindada entre los profesionales que realizan curso presencial, con aquellos que lo realizan por E-leaning, por lo que se comprueba que la incorporación de metodología a distancia, mantiene el nivel de aprendizaje en relación a la metodología presencial, avalando la decisión de realizar la formación de Champions de evidencia a través del uso de cursos a distancia. Esto, a su vez, se encuentra respaldado por los resultados clínicos encontrados en la data de úlceras por presión reportadas en el sistema de eventos adversos entre los años 2014 y 2016.

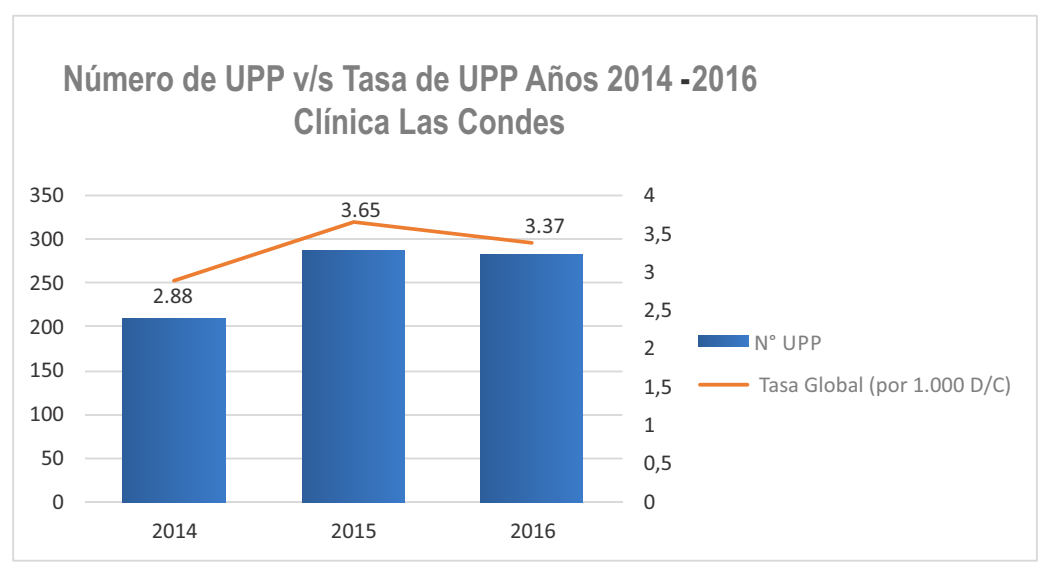

Fuente: Base de datos Gestión de eventos adversos CLC, años 2014-2016.

Figura 5. Número de Úlceras por Presión v/s Tasa de Úlceras por Presión para los años 2015-2016. 
Finalmente, podemos concluir que este sistema permite masificar la formación, llevar control de asistencia, evaluar calificaciones, recibir retroalimentación por parte de los alumnos y hacer seguimiento del aprendizaje obtenido, facilitando el proceso de capacitación y disminuyendo la variabilidad en los cuidados.

Para la implantación de Guías de Práctica Clínica de RNAO en nuestra Institución es y seguirá siendo fundamental el uso de metodologías modernas según los rápidos avances de la tecnología que nos permitan compatibilizar la entrega de contenidos educativos de calidad y el fomento del bienestar de nuestros colaboradores a través de la protección de su tiempo libre.

\section{Conflicto de intereses}

Los autores declaran no tener conflicto de interés.

\section{Financiación}

El desarrollo del curso E-learning fue financiado por la Clínica Las Condes de Chile dentro del marco del Programa de Capacitación del personal.

\section{Referencias}

1. Grinspun D. Guías de práctica clínica y entorno laboral basados en la evidencia elaboradas por la Registered Nurses' Association of Ontario (RNAO). Enferm Clin. 2011; 21(1):1-2. Disponible en: https://doi.org/10.1016/ j.enfcli.2010.11.002.

2. Young $P$, Hortis V, Chambi M, Finn B. Florence Nightingale (1820-1910), a 101 años de su fallecimiento. Rev. méd. Chile. 2011; 139(6):807-13. Disponible en:
http://dx.doi.org/10.4067/S0034-98872011000600017.

3. Registered Nurses' Association of Ontario (RNAO). Clinical Practice Guidelines Program. [Internet]. (2013). [citado 2017 Ene 07]. Disponible en: http://rnao.ca/bpg/ guidelines/clinical.

4. Clínica los Condes. Memorial Anual. [Internet]. (2013). [citado 2017 Ene 07]. Disponible en: http://www.clc.cl/ Dev_CLC/media/Imagenes/PDF/Del\%2015\%20de\%2 Oabril\%20en\%20adelante\%202013/ProspectoCLCv4.p df.

5. Bajnok I, Grinspum D, Lloyd M, McConnell H. Liderando mejoras de calidad mediannte el desarrollo, implementació, y medición de las guías de buenas prácticas de Enfermería. [Internet]. 2015; 17(3):155162. [citado 2017 Ene 07]. Disponible en: http:// revistas.unab.edu.co/index.php?journal=medunab\&pa ge=article $\& o p=$ view $\&$ path $\% 5 B \% 5 D=2382 \&$ path $\% 5 B \%$ $5 \mathrm{D}=2042$.

6. García J, Cepeda A, Méndez S, Carrasco M. La educación a distancia en la formación de recursos humanos en el área de la salud. Perinatol Reprod Hum. 2011; 25(1):46-49.

7. Barrios S, Masalán M, Cook M. Educación en salud: en la búsqueda de metodologías innovadoras. Cienc. Enferm. 2011; 17(1):57-69. Disponible en: http://dx.doi.org/10.4067/S0717-95532011000100007.

8. Grinspun D. Guías de práctica clínica y entorno laboral basados en la evidencia elaboradas por la Registered Nurses' Association of Ontario (RNAO). Enferm Clin. 2011; 21(1):1-2. Disponible en: https://doi.org/ 10.1016/j.enfcli.2010.11.002.

9. Best N, Guideline P. Assessment and Management of Pressure Ulcers. [Internet]. (2005). [citado 2017 Ene 07]. Disponible en : http://rnao.ca/bpg/guidelines/clinical.

10. Best N, Guideline P. Care and Maintenance to Reduce Greetings from Doris Grinspun. [Internet]. (2005). [citado 2017 Ene 19]. Disponible en: http://rnao.ca/bpg/ guidelines/clinical.

11. Grinspun D, Virani T, Bajnok I. Nursing best practice guidelines: The RNAO (Registered Nurses' Association of Ontario) project. Hospital Quarterly. 2002; 5(2):56-60. 